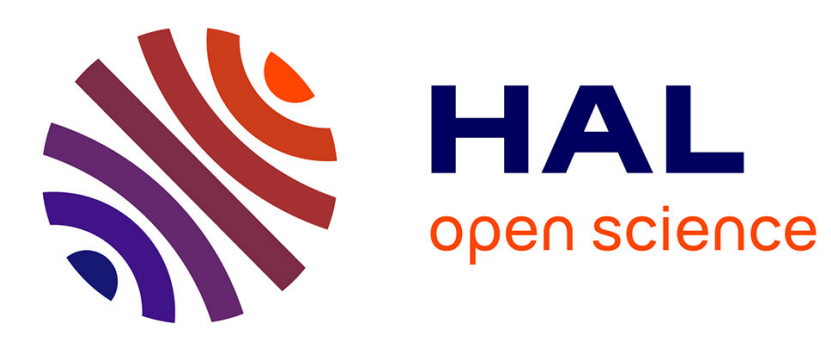

\title{
Spatially resolved phase objects using Mach-Zehnder interferometry
}

V. Besse, C. Cassagne, Georges Boudebs

\section{To cite this version:}

V. Besse, C. Cassagne, Georges Boudebs. Spatially resolved phase objects using Mach-Zehnder interferometry. Journal of Modern Optics, 2012, 59 (10), pp.887-892. 10.1080/09500340.2012.676097 . hal-02442888

\section{HAL Id: hal-02442888 \\ https://hal.science/hal-02442888}

Submitted on 16 Apr 2021

HAL is a multi-disciplinary open access archive for the deposit and dissemination of scientific research documents, whether they are published or not. The documents may come from teaching and research institutions in France or abroad, or from public or private research centers.
L'archive ouverte pluridisciplinaire HAL, est destinée au dépôt et à la diffusion de documents scientifiques de niveau recherche, publiés ou non, émanant des établissements d'enseignement et de recherche français ou étrangers, des laboratoires publics ou privés.

\section{(c)(1)}

Distributed under a Creative Commons Attribution| 4.0 International License 


\title{
Spatially resolved phase objects using Mach-Zehnder interferometry
}

\author{
V. Besse, C. Cassagne and G. Boudebs* \\ LUNAM Université, LPhiA, Laboratoire de Photoniques d'Angers, EA 4464, Université d'Angers, \\ 2 Boulevard Lavoisier, 49045 Angers Cedex 01, France
}

\begin{abstract}
Phase characterization with a good spatial resolution is crucial for focused beams in nonlinear media. The phaseshifting interferometry technique, using the least-squares error criterion for several interferograms, is implemented using a reflective spatial light modulator (SLM). The method provides a convenient calibration for any phase-shift steps. The reliability of the proposed method is checked by direct comparison with results obtained by the Fourier transform method as well as using a previously characterized circular phase object.
\end{abstract}

Keywords: Mach-Zehnder interferometry; Fourier transform; phase object; phase-shifting method

\section{Introduction}

Phase, amplitude and shape characterization of a structure's media are fundamental to many actual research fields. One could use noninvasive interferometric techniques to produce fringe patterns from which we can extract several optical quantities. Different methods exist, such as Twyman-Green interferometry [1], two-beam sharing interferometry [2], shear interferometry [3] and Mach-Zehnder interferometry [4,5]. Actually the automatic analysis of interferograms using digital image processing has become mature and specific applications in the domain of nonlinear photonics remain a subject of active research.

The final goal of our study is to analyze the phase of a laser beam at the output of a nonlinear medium where a nonlinear phase-shift is induced by high laser intensity profile. The Mach-Zehnder interferometer (MZI) combined with a charge-coupled device (CCD) for image processing was proposed in [4]. The main advantage of a MZI when compared to other interference techniques is to provide a large space for imaging systems or materials in both of its arms. With the previously proposed method described in detail in [4] we could obtain intensity-resolved information by comparing the profile of the resolved phase, pixel per pixel, with that of the incident beam. So, it was possible to resolve the spatial profile of a complex nonlinear variation index using the Fourier transform method with only one intense laser shot in the nonlinear material, but the inconvenience was the poor spatial resolution especially when using focused beams. To improve this criterion, the phase-shifting interferometry technique is implemented here using a reflective spatial light modulator (SLM) instead of one mirror. Moreover our method provides a convenient in situ calibration for each individual acquisition. The reliability of the proposed method is checked by direct comparison with results obtained using the Fourier transform method.

\section{Phase-shifting methods (PSM)}

By omitting the time dependency, we can generally express the $n$th interferogram acquired by the CCD:

$$
I_{n}(x, y)=a(x, y)+b(x, y) \cos \left[\Delta \varphi(x, y)+\varphi_{n}\right],
$$

where $(x, y)$ is an arbitrary point in the interference pattern, $n=1,2, \ldots, N$ is an integer, $\varphi_{n}$ is a phase-shift given by the SLM and $\Delta \varphi(x, y)$ is the phase profile to be determined. From this simple expression, which contains three unknown parameters $a(x, y)$ (the background), $b(x, y)$ (the visibility) and $\Delta \varphi(x, y)$, one needs to have at least three equations to find a solution to the problem [6]. In what follows, the $(x, y)$ dependency is sometimes omitted for readability and consistence. One of the advantages of the PSM is the obtained high spatial resolution: the number of measurement points is equal to the number of pixels inside the camera. Moreover, available computing power nowadays allows real time numerical processing of a dozen interferograms using least-squares error criterion [7]. By means of a conventional trigonometric identity, Equation (1) can be rewritten:

$$
I_{n}=a_{0}+a_{1} \cos \left(\varphi_{n}\right)+a_{2} \sin \left(\varphi_{n}\right)
$$


The unknowns are now, $a_{0}=a, a_{1}=b \cos (\Delta \varphi)$ and $a_{2}=b \sin (\Delta \varphi)$. The interferograms must minimize the following squared difference:

$$
\varepsilon=\sum_{n=1}^{N}\left|I_{n}-\left[a_{0}+a_{1} \cos \left(\varphi_{n}\right)+a_{2} \sin \left(\varphi_{n}\right)\right]\right|^{2} .
$$

$\varepsilon$ will be minimum when the partial derivatives of $a_{0}, a_{1}$ and $a_{2}$ are equal to zero:

$$
\frac{\partial \varepsilon}{\partial a_{0}}=\frac{\partial \varepsilon}{\partial a_{1}}=\frac{\partial \varepsilon}{\partial a_{2}}=0 .
$$

The three equations linear system obtained with three unknowns can be written using the matrix notation:

$$
A\left(\varphi_{n}\right) X=B\left(I_{n}, \varphi_{n}\right),
$$

where

$$
\begin{gathered}
X=\left(\begin{array}{l}
a_{0} \\
a_{1} \\
a_{2}
\end{array}\right), \\
A\left(\varphi_{n}\right)=\left(\begin{array}{ccc}
N & \sum_{n=1}^{N} \cos \left(\varphi_{n}\right) & \sum_{n=1}^{N} \sin \left(\varphi_{n}\right) \\
\sum_{n=1}^{N} \cos \left(\varphi_{n}\right) & \sum_{n=1}^{N} \cos ^{2}\left(\varphi_{n}\right) & \sum_{n=1}^{N} \cos \left(\varphi_{n}\right) \sin \left(\varphi_{n}\right) \\
\sum_{n=1}^{N} \sin \left(\varphi_{n}\right) & \sum_{n=1}^{N} \cos \left(\varphi_{n}\right) \sin \left(\varphi_{n}\right) & \sum_{n=1}^{N} \sin ^{2}\left(\varphi_{n}\right)
\end{array}\right)
\end{gathered}
$$

and

$$
B\left(I_{n}, \varphi_{n}\right)=\left(\begin{array}{l}
\sum_{n=1}^{N} I_{n} \\
\sum_{n=1}^{N} I_{n} \cos \left(\varphi_{n}\right) \\
\sum_{n=1}^{N} I_{n} \sin \left(\varphi_{n}\right)
\end{array}\right)
$$

By inverting the matrix system, we can find a solution for $X=A^{-1}\left(\varphi_{n}\right) B\left(I_{n}, \varphi_{n}\right)$, from which the required phase profile is calculated:

$$
\Delta \varphi=\arctan \left(\frac{a_{2}}{a_{1}}\right)
$$

By choosing convenient phase-shifts as in the Carré method [6] (e.g. $\left.\varphi_{n}=(n-1) \varphi=2(n-1) \pi / N\right)$, the A matrix becomes diagonal and the phase profile is found to be:

$$
\left.\Delta \varphi=\arctan \frac{\sum_{n=1}^{N} I_{n} \sin [(n-1) \varphi]}{\sum_{n=1}^{N} I_{n} \cos [(n-1) \varphi]}\right) .
$$

Many formulas have been developed for extracting analytical expressions for $\Delta \varphi$, the phase distribution from the measured intensity images. The Carré, three-step [8] and four-step $[9,10]$ techniques are well known and extensively used in practice. Let us mention here the analytical result for the four-step method:

$$
\Delta \varphi=\arctan \left(\frac{I_{4}-I_{2}}{I_{1}-I_{3}}\right) .
$$

Equal phase-shifts are required and miscalibration of these devices is a source of large phase errors [11]. In the experimental section a convenient calibration for each individual acquisition is proposed, especially to determine the effective values of $\varphi_{n}$.

\section{Fourier-transform method (FTM)}

Takeda et al. proposed the concept of Fourier-transform method of fringe-pattern analysis for computerbased topography and interferometry [12]. The authors conceived a non-contact profilometer [13] with this method. It used initially one-dimensional FFT algorithms, and then it evolved to two-dimensional global form with the advent of fast algorithms FFT2. One of the most important properties is that Fouriertransform is a global operation. This method has the great advantage of considerably reducing the noise level of the phase profile, but degrades the spatial resolution. This property will be discussed hereafter according to our results.

Experimentally, it is easy to obtain rectilinear fringes. Optimally the size of the fringes should be four times larger than that of the sampling pixel in order to optimize the spatial resolution of the phase profile. The acquired interference pattern can be written:

$$
I(x, y)=a(x, y)+b(x, y) \cos \left[\Delta \varphi(x, y)+2 \pi f_{0} x\right],
$$

where $a(x, y)$ and $b(x, y)$ represent the low-frequency background intensity and the fringe amplitude (visibility), respectively. $\Delta \varphi(x, y)$ is the phase profile to be determined and $f_{0}$ represents the spatial carrier frequency of the interference pattern. To understand physically how this method operates, we rewrite this equation using complex notation:

$$
\begin{aligned}
I(x, y)= & a(x, y)+c(x, y) \exp \left(2 j \pi f_{0} x\right) \\
& +c^{*}(x, y) \exp \left(-2 j \pi f_{0} x\right),
\end{aligned}
$$

where we have defined $c(x, y)=b(x, y) \exp [i \Delta \varphi(x, y)] / 2$ and $c^{*}$ indicates the complex conjugate. 
Next, we apply a Fourier transform to Equation (10) to give:

$$
\tilde{I}\left(f_{x}, f_{y}\right)=\tilde{A}\left(f_{x}, f_{y}\right)+\tilde{C}\left(f_{x}-f_{0}, f_{y}\right)+\tilde{C}^{*}\left(f_{x}+f_{0}, f_{y}\right),
$$

where $\tilde{A}\left(f_{x}, f_{y}\right)=T F[a(x, y)], \quad \tilde{C}\left(f_{x}, f_{y}\right)=T F[c(x, y)]$ and $f_{x}, f_{y}$ are the spatial frequency in the $\mathrm{x}$ and $\mathrm{y}$ directions, respectively. The spectrum $\widetilde{I}$ is nonzero around three regions: $\widetilde{A}$, the central area representing the background and two other symmetrical areas located at $\pm f_{0}$, the spatial frequencies of the cosine function. So one has to choose $f_{0}$ conveniently, large enough in order not to have spectral overlapping between the components in Equation (11), and sufficiently low to let one fringe cover at least $4 \mathrm{CCD}$ pixels. By using a rectangular window function centered at $\mathrm{f}_{0}$ we can extract $\widetilde{C}$ from $\widetilde{I}$. Then after performing a translation to the origin we proceed with an inverse Fourier transform:

$$
c(x, y)=\frac{1}{2} b(x, y) \exp [j \Delta \varphi(x, y)],
$$

from which we deduce the optical phase by:

$$
\Delta \varphi(x, y)=\arctan \left\{\frac{\operatorname{Im}[c(x, y)]}{\operatorname{Re}[c(x, y)]}\right\} .
$$

The phase profile is unwrapped at $2 \pi$ phase discontinuities to recover a continuous phase distribution. For a given wavelength $\lambda$, it is possible to express the height distribution $h(x, y)$ if the refractive index $n$ of the medium is known:

$$
h(x, y)=\frac{\lambda \Delta \varphi(x, y)}{2 \pi(n-1)} .
$$

\section{Experimental setup}

The built MZI is illuminated with a $\mathrm{He}-\mathrm{Ne}$ laser emitting at $632.8 \mathrm{~nm}$ (see Figure 1). The first two lenses and the pinhole at the middle play the role of spatial filter to clean the beam. Then the collimated beam is divided into a test beam passing through the phase object (PO) to analyze and a reference beam where the mirror is replaced by a reflective spatial light modulator (SLM). The SLM is based on a reflective Liquid crystal on silicon micro-displays with $8 \mu \mathrm{m}$ pitch, $1920 \times 1080$ pixels resolution and is optimized to give a phase-shift above $2 \pi$ in the visible range with a maximum of $3.7 \pi$ at $\lambda=632 \mathrm{~nm}$. It is controlled by a software interface allowing the user to select a matrix level gray easy to transform via a graphic card into a matrix phase-shift. A $1388 \times 1038$ pixels CCD camera $(6.45 \mu \mathrm{m}$ pixel size) is placed at the MZI output.
Generally, the final lens is placed at two times its focal length to form an image of the PO at the CCD but it is possible to have higher magnification for enhanced spatial resolution modifying the image and object distances to the lens.

The phase-changing circular plate consists of a glass structure on which a transparent dielectric disc (of radius $L_{p}=0.5 \mathrm{~mm}$ ) has been deposited (Figure 1(b)). The disc has a thickness and index of refraction such that it retards the phase of the incident light by $\pi$ radians relative to the phase outside the disc at $1064 \mathrm{~nm}$. The PO serves as a test object; it was previously used in a $4 \mathrm{f}$ system for nonlinear refractive index measurement [14]. Theoretically, in our experiment (red $\mathrm{HeNe}$ laser) the phase-shift should be around: $632 \pi / 1064 \simeq 1.8 \mathrm{rad}$.

\section{Phase-shift calibration and measurement process}

\subsection{PSM}

The SLM provides a phase-shift which is not linear versus the gray scale applied to its pixels (Figure 2(a)). It is necessary to calibrate the system and preferably for each individual experiment in PSM. For this purpose we have developed an algorithm to measure the displacement of the fringe profile whatever the applied phase-shift, $\varphi_{n}$. The setup is aligned in order to obtain linear fringes at the CCD. To determine the fringe spacing, $i$, precisely one has to fit the upper part of the bright fringes using a quadratic polynomial expansion. An image profile is displayed on the first acquisition over $\Delta$, a perpendicular axis to the fringes. By determining two maximum positions, $x_{i} \equiv x_{1}$ and $x_{f}$ separated by $n_{0}$, a large number of dark fringes, $i=\left|x_{f}-x_{i}\right| n_{0}$, are observed. The first position $\left(x_{1}\right)$ serves as a $i=\left|x_{f}-x_{i}\right| n_{0}$ reference for the other $\varphi_{n}$ to find out. Next, we load the $n-1$ following acquisitions defining the interferograms of Equation (1) which are given for the unknown phase-shift, $\varphi_{n}$. By clicking again to fit the upper part of the first shifted bright fringe we can easily determine $x_{2}$, the new maximum position where $\varphi_{2}=2 \pi\left(x_{2}-x_{1}\right) / i$. This operation is repeated again over all the remaining acquisitions defining the $\varphi_{n}$ values (see Figure 2(b)). Note that a calibration could be done once for all, as it is shown by Figure 2(a) where we have plotted the ratio of the obtained phase-shift by $2 \pi$ versus the SLM gray levels (corresponding to 256 phase levels) generated by the software. We have preferred to define precisely the phase-shift value for each acquisition because the SLM is sensitive to different parameters such as light polarization, wavelength and mechanical vibration of the optical breadboard. Matrix $\mathrm{A}\left(\varphi_{n}\right)$ (Equation (5)) can be constructed, inverted and the solution $\mathrm{X}$ can be 
(a)

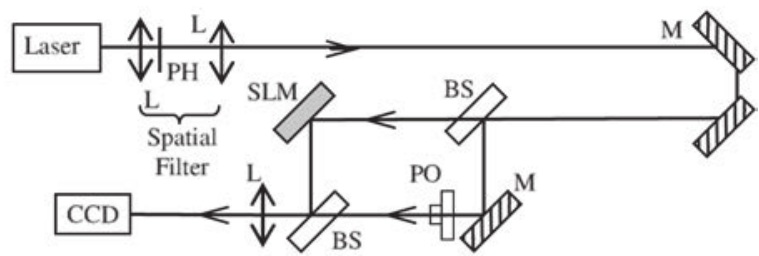

(b)

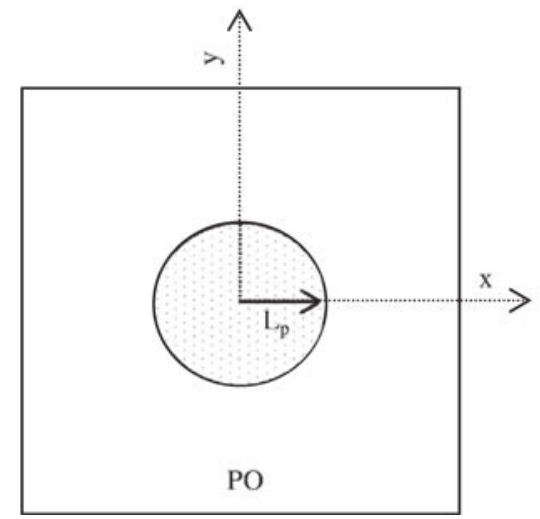

Figure 1. (a) Experimental setup. PO: phase object; L: lenses; M: mirrors; BS: beam splitters; PH: pin hole; SLM: spatial light modulator. (b) Schematic of the PO, the circular phase object.

quickly obtained. Equation (6) is calculated as wrapped values lying in the range $-\pi$ to $+\pi$. For this reason a two-dimensional unwrapping step is needed to remove the $2 \pi$ phase jumps. At this level of processing, it was necessary to implement a real-time correction function. As can be seen in Figure 3, one of the interferograms showed several points due to dust on the PO where singularities could appear. By mouse clicking on these discontinuities a local convolution with a rectangular window is performed over all the interferograms, smoothing the recorded intensities. The window width is a parameter chosen by the operator, but generally 2 pixels are large enough to give convenient results and to eliminate the singularities that may appear. Figure 3 shows $\Delta$, the perpendicular axis to the fringes necessary to recover the phase steps.

The image of the phase object obtained after numerically processing the $N$ fringe patterns (here $N=5)$ is given in Figure $4(a)$. The phase when unwrapped shows a quasi-linear variation going from -5 (lower left corner) to $15 \mathrm{rad}$ (upper right corner) where the PO is added on. The linear variation is represented by a base-plane and it is the consequence of the rectilinear fringes alignment. In Figure 4(b), the linear fitting of the base-plane obtained by removing a rectangular window containing the circular $\mathrm{PO}$ is represented. $\Delta \varphi$, the phase distribution, is the difference between the original unwrapped phase (shown in
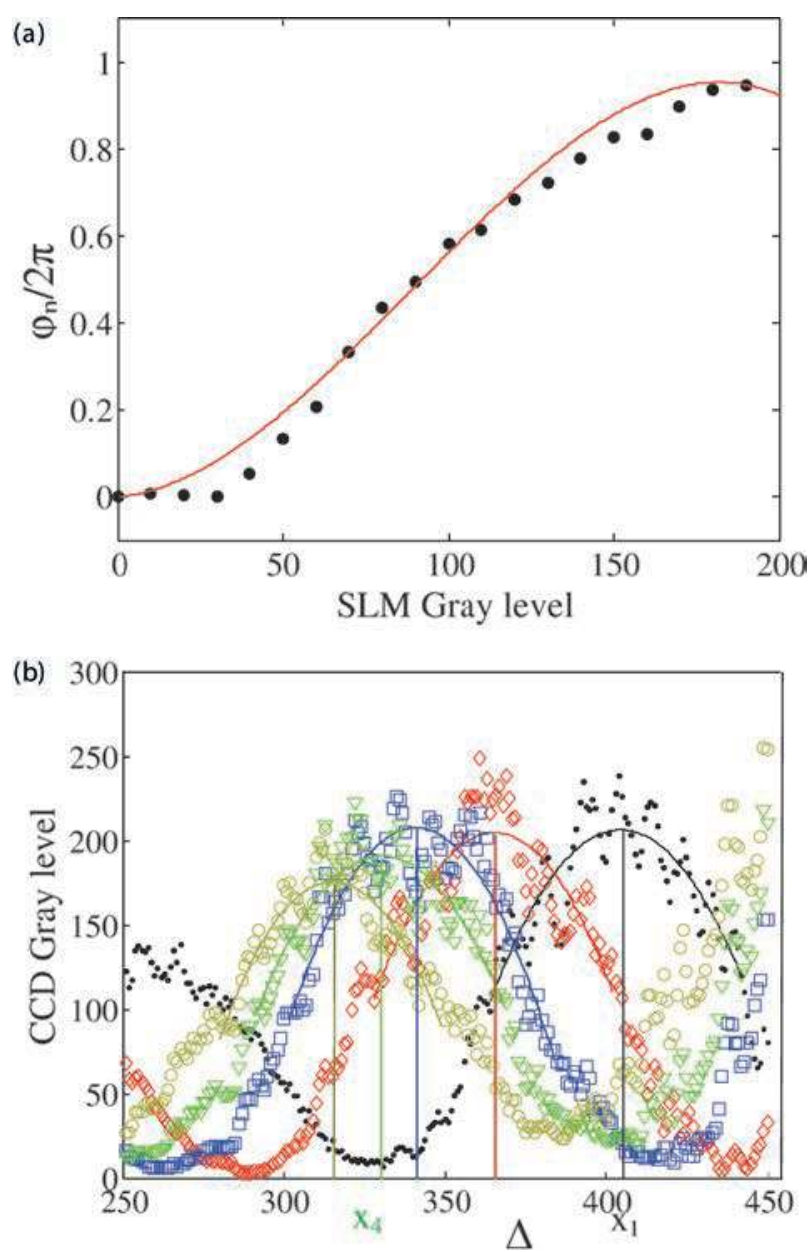

Figure 2. (a) SLM calibration: the ratio of the obtained phase-shift by $2 \pi$ versus the SLM gray levels defined by the software; (b) five experimental fringe profiles (dots, diamonds, squares, triangles, circles) indicating different phaseshifts of the SLM. The phase-shifting values are calculated using the difference between the maximums given by quadratic fitting (solid lines). $\Delta$ is an axis perpendicular to the fringe, as shown in Figure 3. Only $x_{1}$ and $x_{4}$ (two maximum positions) are shown for readability. (The color version of this figure is included in the online version of the journal.)

Figure $4(a)$ ) and the fitted base-plane phase (shown in Figure 4(b)). In Figure 4(c) we can see the result for the PO, i.e. the local phase distribution, after subtraction. Finally, in Figure $4(d)$, the central profile of the previous result is plotted along the $x$ direction. Note that the mean value of the retrieved $\Delta \varphi$ is equal to the expected value (1.8 rad), thus validating our numerical procedure. In order to increase the accuracy of the measurement, the number of the recorded images can be higher than five (taken for this paper). In our example, the time processing was about $10 \mathrm{~s}$ using Matlab computing language. 


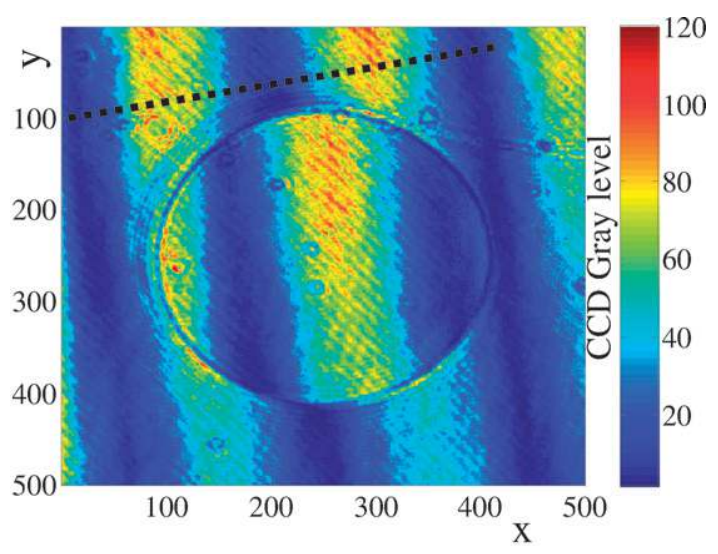

Figure 3. Image of the fringe pattern showing the phase-shift through the transparent phase object. The dashed line in the upper left side represents the $\Delta$ axis perpendicular to the fringes chosen by the operator for in situ calibration and space fringe measurement, as is done in Figure $2(b)$. The vertical bar represents the CCD gray level. (The color version of this figure is included in the online version of the journal.) (a)

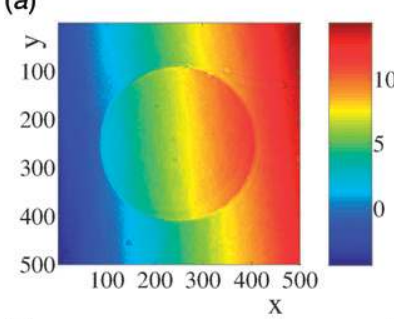

(c)

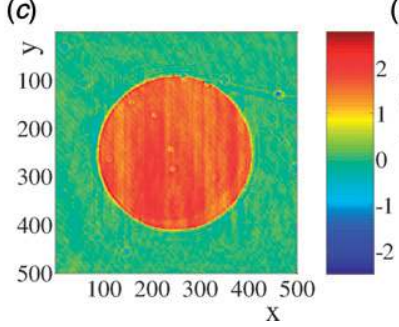

(b)

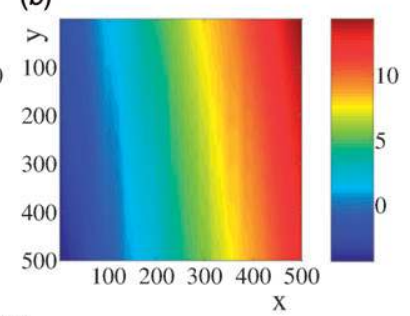

(d)

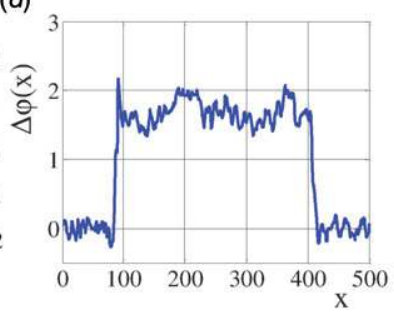

Figure 4. (a) Image of the phase object obtained after numerically processing the $N$ fringe patterns (here $N=5$ ); (b) image of the phase base-plane obtained by linear fitting of the remaining phase in $(a)$ after taking out a rectangular window containing the circular PO; $(c)$ result of the difference between phase values obtained in $(a)$ and $(b)$; the vertical bars indicate the phase in radians; $(d)$ central profile of the phase distribution $\Delta \varphi$ in the $\mathrm{PO}$ along the $x$ direction. (The color version of this figure is included in the online version of the journal.)

\subsection{FTM}

Theoretically one image is needed to retrieve the spatial distribution of the PO [15], but in practice several images are acquired to this purpose. First, the interference patterns are recorded with and without the PO. Figure 5(a) shows the interference pattern in the (a)

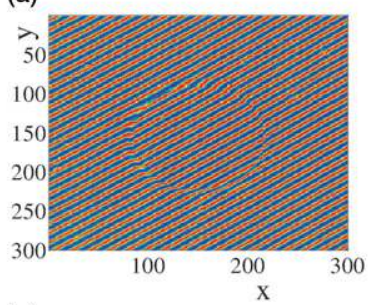

(c)

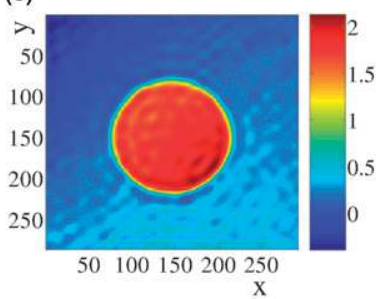

(b)

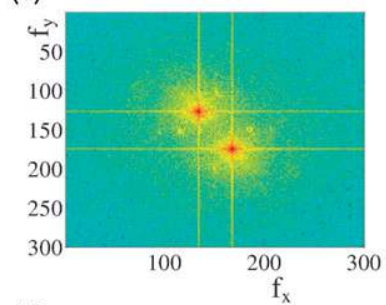

(d)

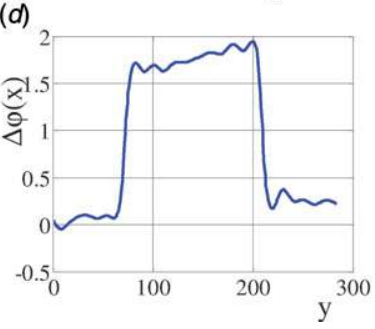

Figure 5. (a) Acquired image of the interference pattern where the sharp deviations of the linear fringes indicate the presence of the PO edge; $(b)$ base 10 logarithm of the spectrum absolute value corresponding to the fringes given in (a); (c) phase retrieving by inverse Fourier transform on a suitable selected area in the spectrum translated to the origin (the vertical bar indicates the phase in radians); $(d)$ central section of the phase distribution $\Delta \varphi$ in $\mathrm{PO}$ along the $x$ direction. (The color version of this figure is included in the online version of the journal.)

presence of the PO where relatively sharp deviations of the linear fringes appear at its circular edge. Then the images given by the test and the reference arms are acquired separately. These patterns are necessary to normalize the interferogram and to reduce the background spatial extension increasing by this way the rectangular window of the spatial filtering process in the Fourier domain. Equation (9) easily leads to:

$$
\begin{aligned}
\cos & {\left[\Delta \varphi(x, y)+2 \pi f_{0} x\right] } \\
= & \frac{I(x, y)-\left[I_{\text {ref }}(x, y)+I_{\text {test }}(x, y)\right]}{2 \sqrt{I_{\text {ref }} \cdot I_{\text {test }}}},
\end{aligned}
$$

where $\quad I_{\text {ref }}(x, y)+I_{\text {test }}(x, y)=a(x, y), \quad b(x, y)=$ $2 \sqrt{I_{\text {ref }}(x, y) \cdot I_{\text {test }}(x, y)}, I_{\text {ref }}$ is the reference arm image alone and $I_{\text {test }}$ is the test arm image with the phase object. To retrieve $\Delta \varphi(x, y)$, a direct fast Fourier transform is applied to the result of the operation given by Equation (15). By using a rectangular window function centered at $f_{0}$ we select the values of the complex spectrum signal inside. Then, the spectrum is centered in a zero matrix having a dimension identical to the initial interference pattern. In Figure 5(b), base 10 logarithm of the absolute value corresponding to the spectrum of the fringes appearing in Figure 5(a) is shown. One can see that the central term representing the background is negligible. Thus, one of the advantages here is to avoid overlapping between the areas around $\pm f_{0}$, the spatial frequency of the fringe spacing 
and the central one (not visible here due to normalization according to Equation (15)). Note that the logarithm is used to enhance the visibility of the lower value in the spectrum. After translation to the origin inside the zero matrix, the phase retrieved by inverse Fourier transform on a suitably selected area in the spectrum is processed. The same numerical processing is done with a reference interferogram (without PO) in order to obtain the image base plane of the phase due to the alignment of the MZI showing the rectilinear fringes (physically to retrieve $f_{0}$ ). Finally, we have to evaluate the ratio between these last two operations to extract and to fully characterize the PO (Figure 5(c)). A central section of the phase distribution $\Delta \varphi$ along the $x$ direction is shown in Figure $5(d)$, where we find the mean value of the phase step to be around $1.8 \mathrm{rad}$, as expected previously.

A comparison of the results given by the phase distributions shown in Figures 4(c) and 5(c) shows that the spatial resolution is higher with the PSM when compared to the FTM. The spatial filtering process is responsible of this inconvenience. One can see very small dust particles on the PO when using PSM (Figure $4(c)$ ). The unwrapping discontinuities can be a problem but the local correction with a real time convolution provided a good solution, especially that singularities do not affect all the details in the final image. The overall aspect of the retrieved phase is more convincing. We are planning to implement the PSM for vortex soliton characterization shaped by the SLM using pulsed laser light at $1064 \mathrm{~nm}$ in the picosecond regime.

\section{Conclusion}

To improve the spatial resolution of an input focused beam in nonlinear media, the phase-shifting interferometery technique using the least-square error criterion was implemented with a reflective SLM inside a Mach-Zehnder configuration. Moreover, we showed that our method provides a convenient in situ calibration for each individual acquisition, avoiding any calibration procedure. The reliability and the validity of the method were checked by direct comparison with results obtained using the Fourier transform method and a previously characterized circular phase object.

\section{References}

[1] Chen, Y.-L.; Hsieh, H.-C.; Wu, W.-T.; Chang, W.-Y.; Su, D.-C. Appl. Opt. 2010, 49, 6888-6892.

[2] De Nicola, S.; Ferraro, P.; Finizio, A.; Pierattini, G. Appl. Opt. 2001, 40, 1631-1636.

[3] Falldorf, C. J. Opt. Soc. Am. A 2011, 28, 1636-1647.

[4] Boudebs, G.; Chis, M.; Nguyen Phu, X. J. Opt. Soc. Am. B 2001, 18, 623-627.

[5] Kupka, D.; Schlup, P.; Bartels, R.A. Appl. Opt. 2008, 47, 1200-1205.

[6] Carre, P. Metrologia 1966, 2, 13-23.

[7] Greivenkamp, J.E. Opt. Eng. 1984, 23, 350-352.

[8] Wyant, J.C.; Koliopoulos, C.L.; Bhushan, B.; George, O.E. ASLE Trans. 1984, 27, 101-113.

[9] Wyant, J.C. Laser Focus (Newton, Mass.) 1982, 18, 65-71.

[10] Schwider, J.; Burow, R.; Elssner, K.E.; Grzanna, J.; Spolaczyk, R.; Merkel, K. Appl. Opt. 1983, 22, 3421-3432.

[11] Takeda, M.; Ina, H.; Kobayashi, S. J. Opt. Soc. Am. 1982, 72, 156-160.

[12] Takeda, M.; Mutoh, K. Appl. Opt. 1983, 22, 3977-3982.

[13] Boudebs, G.; Cherukulappurath, S. Phys. Rev. A: At., Mol., Opt. Phys. 2004, 69, 053813.

[14] Kuang, D.; Han, M.; Gao, H.; Du, Z. J. Opt. (Bristol, U.K.) 2011, 13, 035501. 